FOLIA

Amazónica

Revista del Instituto de Investigaciones

de la Amazonía Peruana

\title{
MONITOREO DE LA ABUNDANCIA DE GUACAMAYOS DURANTE DIEZ AÑOS EN LA CUENCA MEDIA DEL RÍO SAMIRIA, LORETO, PERÚ
}

\author{
Segundo Artidoro ORBE ALVAN ${ }^{1 *}$, Jhon Jairo LÓPEZ-ROJAS², \\ Richard E. BODMER ${ }^{3,4}$, Carlos Marcial LÓPEZ ROJAS ${ }^{5}$
}

1 Universidad Científica del Perú, Facultad de Ciencias e Ingeniería. Av. Abelardo Quiñones km 2,5, San Juan Bautista, Loreto, Perú.

2 Estación Experimental Agraria El Porvenir. Dirección de Desarrollo Tecnológico Agrario, Instituto Nacional de Innovación Agraria (INIA), Jr. Martinez de Compagñon 1035, Tarapoto, San Martín 22200, Perú.

3 Durrell Institute of Conservation and Ecology, School of Anthropology and Conservation, University of Kent, Canterbury, Kent, UK.

4 Museum of Indigenous Amazonian Cultures, Fundamazonia, 332 Malecón Tarapacá, Iquitos, Loreto, Perú.

5 Dirección Ejecutiva de Administración y Conservación de los Recursos Naturales, Autoridad Regional Ambiental (ARA), Moyobamba, Perú.

* Correo electrónico: segundozorbe@gmail.com

\section{RESUMEN}

Los cambios climáticos que se han producido en las últimas décadas han influenciado en las tendencias y fluctuaciones poblacionales de muchas especies amazónicas, incluyendo los guacamayos. Entre 2006 y 2015, se cuantificaron guacamayos en la cuenca media del río Samiria en Perú. Los conteos por puntos distribuidos a lo largo de ocho transectos acuáticos en los sectores de Tacshacocha y Huishtococha se aplicaron durante los períodos de creciente y vaciante del río, para determinar la abundancia de guacamayos y los posibles cambios debido a eventos climáticos. Un total de 4225 pseudoréplicas en 72 estaciones de puntos de conteo resultaron en 65149 avistamientos de guacamayos. Registramos cinco especies: Orthopsittaca manilatus, Ara ararauna, Ara severus, Ara chloropterus y Ara macao. Los resultados mostraron una alta consistencia durante los diez años, siendo Orthopsittaca manilatus la especie más abundante y, Ara chloropterus y 
Ara macao, entre las especies más raras; ambos grupos muestran diferencias entre Tacshacocha y Huishtococha. La población de los guacamayos fluctuó a lo largo de los años. Las especies Ara ararauna, Ara severus y Ara macao, tuvieron una disminución en sus poblaciones, sin embargo, esta tendencia no fue significativa y sus poblaciones se han mantenido estables durante la última década. Orthopsittaca manilatus y Ara chloropterus fueron las únicas especies cuyas poblaciones han aumentado en comparación con las evidencias de clima seco generadas en el 2010, debido a la estacionalidad de los niveles de agua del río Marañón y Amazonas.

PALABRAS CLAVE: eventos climáticos, nivel del agua de río, Psittacidae, Reserva Nacional Pacaya Samiria, tendencia poblacional.

\title{
MONITORING THE ABUNDANCE OF MACAWS FOR TEN YEARS IN THE MIDDLE BASIN OF THE SAMIRIA RIVER, LORETO, PERU
}

\begin{abstract}
The climatic changes that have occurred in the last decades have influenced the trends and population fluctuations of many Amazonian species, including the macaws. Between 2006 and 2015, macaws were quantified on the mid watershed of the Samiria river in Peru. Point counts distributed along 8 aquatic transects on the Tacshacocha and Huishtococha sectors were applied during growing and dry seasons to determine macaw abundance and the possible changes due to climatic events. With a total of 4225 pseudo-replications in 72 counting point stations, there were 65149 macaw sightings. We recorded five species: Orthopsittaca manilatus, Ara ararauna, Ara severus, Ara chloropterus and Ara macao. The result displayed high consistency during the ten years, with $O$. manilatus being the most abundant species and the rarest species $A$. chloropterus and A. macao; with both groups showing differences between the Tacshacocha and Huishtococha. The macaw population fluctuated throughout the years. The species $A$. ararauna, $A$. severus and $A$. macao had a decline in their populations, yetthis trend was not significant and their populations have remained stable during the last decade. Orthopsittaca manilatus and Ara chloropterus are the only species whose populations have increased with indications of the dry generated in 2010 due to the seasonality of the Marañon and Amazonas river water levels.

KEYWORDS: Climatic events, river water level, Psittacidae, Pacaya Samiria National Reserve, Population trend.
\end{abstract}




\section{INTRODUCCIÓN}

Los guacamayos son un grupo de aves de la familia Psittacidae, del orden Psittaciformes que se distribuyen aproximadamente desde las selvas mexicanas hasta el noreste de Argentina. Se reconocen 17 especies, divididos en seis géneros. En el Perú, existen cuatro géneros: Ara, Diopsitacca, Orthopsittaca y Primolius que comprenden ocho especies en total (Plenge, 2020); cinco de esas especies se encuentran en la Reserva Nacional Pacaya Samiria (RNPS): Ara ararauna Linnaeus 1758, A. chloropterus Gray 1859, A. macao Linnaeus 1758, A. severus Linnaeus 1758 y Orthopsittaca manilatus Boddaert 1783 (Bodmer et al., 2014). Estas especies están consideradas dentro de la categoría de preocupación menor, con tendencia poblacional estable a decreciente (IUCN, 2020) e incluidas en los apéndices I y II de CITES (CITES, 2020).

La RNPS se encuentra influenciada por importantes fluctuaciones estacionales (creciente y vaciante), causadas por el pulso de inundación de los ríos amazónicos que influyen en la ecología de la vida silvestre (Bodmer et al., 2006). Por ejemplo, los guacamayos tienen una alta dependencia a la fructificación estacional de los aguajales Mauritia flexuosa (Bodmer et al., 2006; Silva \& Melo, 2015; Hosein et al., 2017).

Es importante mencionar que durante las últimas décadas la cuenca del Amazonas ha sido escenario de sequías e inundaciones extremas (Gloor et al., 2013; Espinoza et al., 2014; Marengo \& Espinoza, 2015) con impactos positivos y negativos en la biodiversidad (Zulkafli et al., 2015; Bodmer et al., 2017), influyendo en el equilibro ecológico entre la pérdida de especies sensibles y la tendencia a aumentar o permanecer estables en especies tolerantes (Costa et al., 2020). Por ejemplo, Bodmer et al. (2014) mostraron que la fluctuación temporal de algunas especies de guacamayos en el río Samiria, entre 2006 y 2012, no se vio afectada por las condiciones de sequía en la Amazonía durante el 2010.

En base a ello, nos preguntamos si ocurrieron cambios en la abundancia de guacamayos debido a la existencia de fluctuaciones del nivel del río Samiria. Para eso, la presente investigación tiene el propósito de determinar la existencia de variación estacional en la abundancia de las especies de guacamayos durante diez años en la cuenca media del río Samiria, y si estos cambios están relacionados con la presencia de eventos climáticos extremos ocurridos durante la última década.

\section{MATERIAL Y MÉTODOS}

\section{ÁREA DE ESTUDIO}

Este estudio se realizó en la cuenca media del río Samiria (453'28,79"S; $74^{\circ} 25^{\prime} 52,60^{\prime \prime} 0$ ), en la Reserva Nacional Pacaya Samiria, Loreto, Perú. Esta zona presenta un bosque húmedo tropical con una temperatura promedio anual que varía de $20,1{ }^{\circ} \mathrm{C}$ a $33,1{ }^{\circ} \mathrm{C}$ y con una precipitación promedio anual de 2000 a $3000 \mathrm{~mm}$. Por tanto, la precipitación se distribuye de tal manera que no hay una estación seca bien definida y prevalece un alto porcentaje de humedad (80-94\%) (Bayley et al., 1992).

Se establecieron dos sectores de muestreo en la cuenca media del río Samiria: Tacshacocha (453'11.24"S; 74²1'14.21"0, $118 \mathrm{~m}$ ) y Huishtococha $\quad 4^{\circ} 54^{\prime} 46,70^{\prime \prime S} ; \quad 74^{\circ} 28^{\prime} 14,37^{\prime \prime} \mathrm{O}$; $117 \mathrm{~m})$. Se recorrieron cuatro transectos en cada uno de los sectores (Figura 1), en el caso de Tacshacocha se recorrieron: río abajo (Río 1), río arriba (Río 2), un canal de conexión (Canal 1) y el lago Atún (Lago 1); en el caso del sector de Huishtococha se recorrieron: río abajo (Río 3), río arriba (Río 4), un canal de conexión (Canal 2) y el lago Huishto (Lago 2). Los muestreos se realizaron estacionalmente: vaciante (todos 


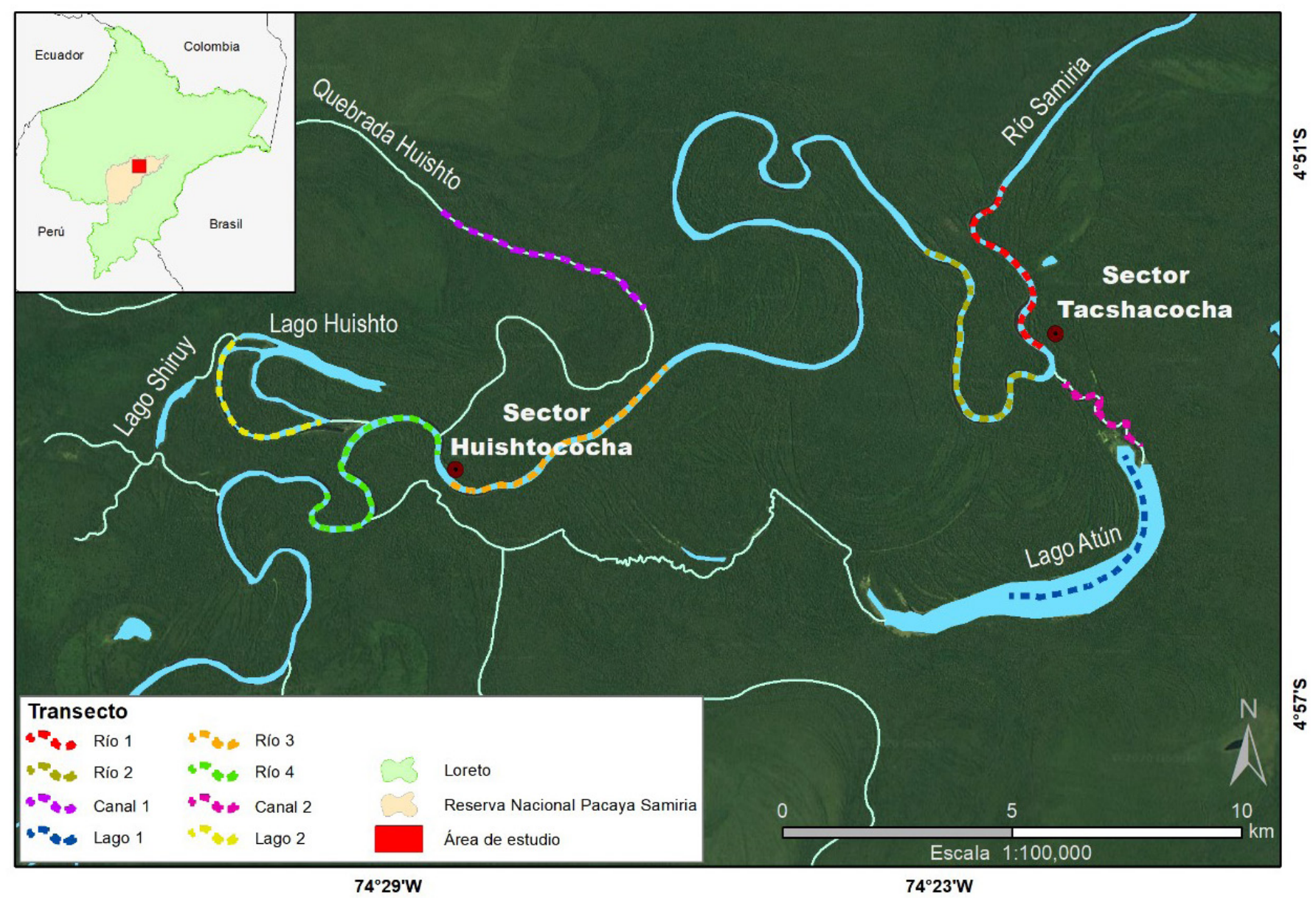

Figura 1. Mapa de ubicación de los transectos por sectores para el censo de guacamayos en la cuenca media del río Samiria (Loreto, Perú).

los días durante los meses de mayo a agosto) y creciente (cada dos semanas de noviembre a marzo). Se aplicó la metodología de conteos por puntos, para ello, se recorrieron los transectos de 4-4,5 km por las mañanas (5:30-9:00 h) y de 3-3,5 km por las tardes (16:00-18:30 h). Cada punto de muestreo estuvo separado por $500 \mathrm{~m}$, con un tiempo de 15 minutos por punto de avistamiento. Se usó un pequeño bote de madera motorizado, un dispositivo GPS para medir la distancia entre puntos, ilustraciones de especies de guacamayos y binoculares para las identificaciones de guacamayos. Se cuantificaron gran parte de los guacamayos al vuelo $\mathrm{y}$ esporádicamente individuos posados.
Se utilizaron datos colectados desde 2006 y 2015 en la cuenca media del río Samiria por la Fundación Latinoamericana para el Trópico Amazónico (FUNDAMAZONIA). Estos fueron colectados con el mismo método antes señalado, con la finalidad de estandarizar la información histórica.

Se obtuvo la base de datos históricos del nivel del río Amazonas entre los años 2006 al 2015 del Servicio Nacional de Meteorología e Hidrología del Perú (SENAMHI, 2020), con la premisa que el aumento o disminución del caudal afecta a todos los ríos que desembocan en esta parte de la Amazonía. 


\section{ANÁLISIS DE DATOS}

La abundancia de guacamayos fue estimada con el índice de abundancia (ind./punto). Se consideró la mediana como medida de tendencia central y al rango intercuartílico (RIQ) como medida de precisión. Para determinar los transectos más importantes para las especies de guacamayos se consideró un mínimo de 50 \% de explicación de los componentes I y II del análisis de componentes principales (PCA) con una matriz de covarianza. Las relaciones entre las abundancias de las especies fueron realizadas con la correlación múltiple de Pearson. La tendencia poblacional de guacamayos fue estimada con la regresión lineal mediante a la interpretación del slope y su probabilidad.

Los cambios en la comunidad de guacamayos fueron evaluados mediante un análisis de similaridad (ANOSIM) en una PCA para diferenciar el antes y después de la vaciante del 2010. Todos los datos fueron analizados y graficados en el software RStudio (RStudio Team, 2019) y Sigmaplot 14.0 (Systat, 2017).

\section{RESULTADOS}

Se obtuvo 65149 avistamientos de guacamayos en 72 estaciones de puntos de conteo (con un total 4225 pseudoréplicas), distribuidos en ocho transectos de Tacshacocha y Huishtococha. La abundancia de guacamayos fueron diferentes entre las especies $(\mathrm{H}=32,07 ; \mathrm{gl}=4 ; p<0,001)$. La especie más abundante ( $50 \%$ de la muestra) fue Orthopsittaca manilatus, con una mediana de 5,9 ind./punto (RIQ = 5,1 a 13,9 ind./punto) (Figura 2). La segunda especie más abundante fue Ara ararauna (mediana = 3,5 ind./punto; RIQ = 2,1 a 4,1 ind./punto), seguida de $A$. severus (mediana $=1,7$ ind./punto; RIQ $=0,8$ a 3,7). Las especies menos abundantes o más raras fueron A. chloropterus (mediana $=0,08$ ind./punto, RIQ $=0,05$ a 0,17 ind./punto) y A. macao (mediana $=$ 0,03 ind./punto; RIQ = 0,002 a 0,11 ind./punto) (Figura 2).

El análisis de componentes principales mostró una diferenciación entre las especies de mayor y menor abundancia y los transectos. El primer

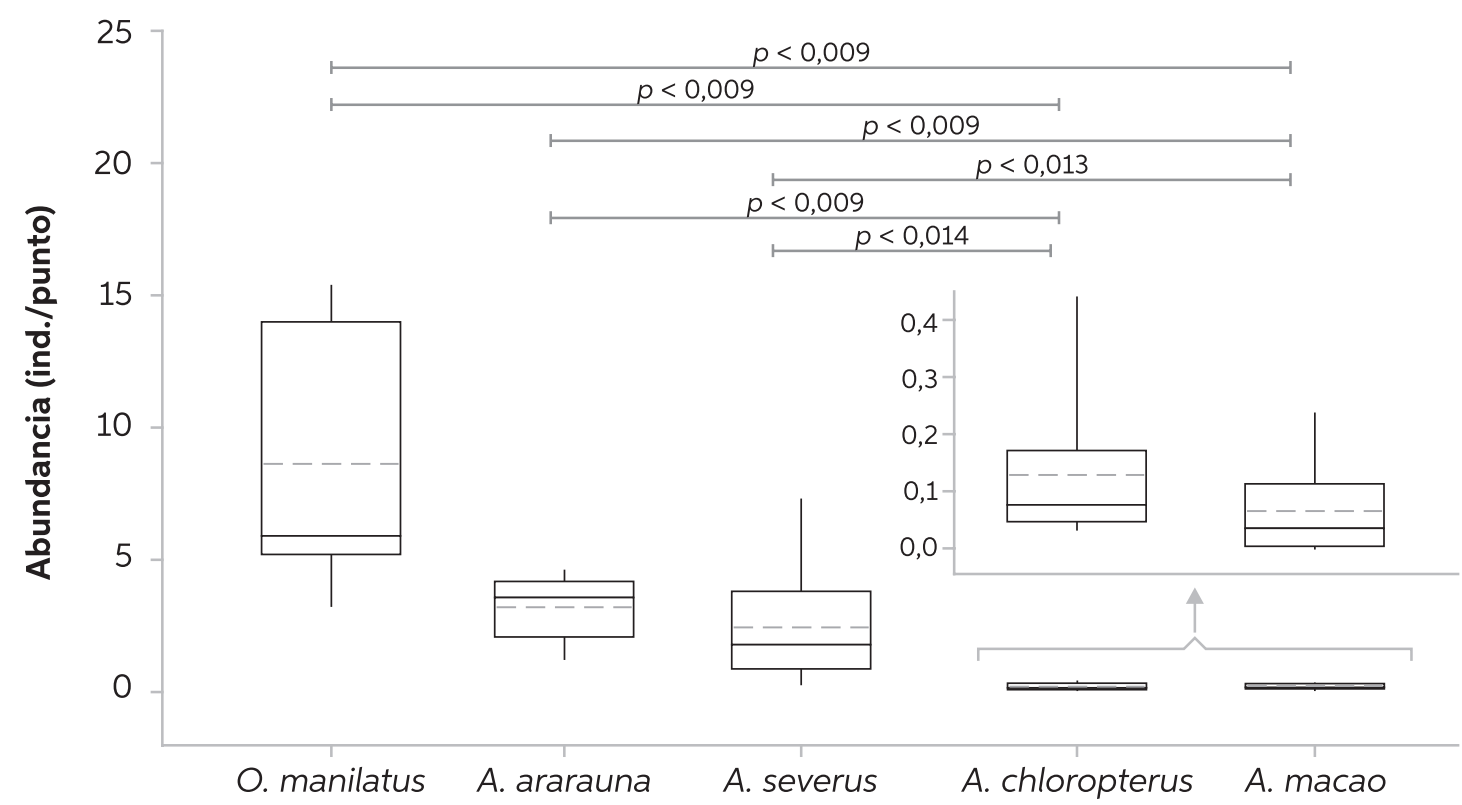

Figura 2. Abundancia de guacamayos en la cuenca media del río Samiria (Loreto, Perú). En los boxplots la línea media indica la mediana, la línea punteada indica la media y la caja el rango intercuartílico. Las líneas por encima de los boxplots, indican la diferencia significativa entre las especies. 
componente tuvo una explicación de 91,19\%, para el caso de $O$. manilatus, la variable más importante que explicó su variación fue Río 1 con un valor de $-0,57$, seguido de la variable Canal 1 con $-0,53$, y la variable Lago 1 con $-0,48$, todos en el sector de Tacshacocha. El segundo componente tuvo un porcentaje de explicación de 7,76\%, y la variable más importante fue el Río 3 , con $-0,88$, el cual explicó la abundancia de $A$. ararauna y $A$. severus en el sector Huishtococha (Figura 3). Mientras que las especies raras $A$. macao y A. chloropterus no mostraron evidencia

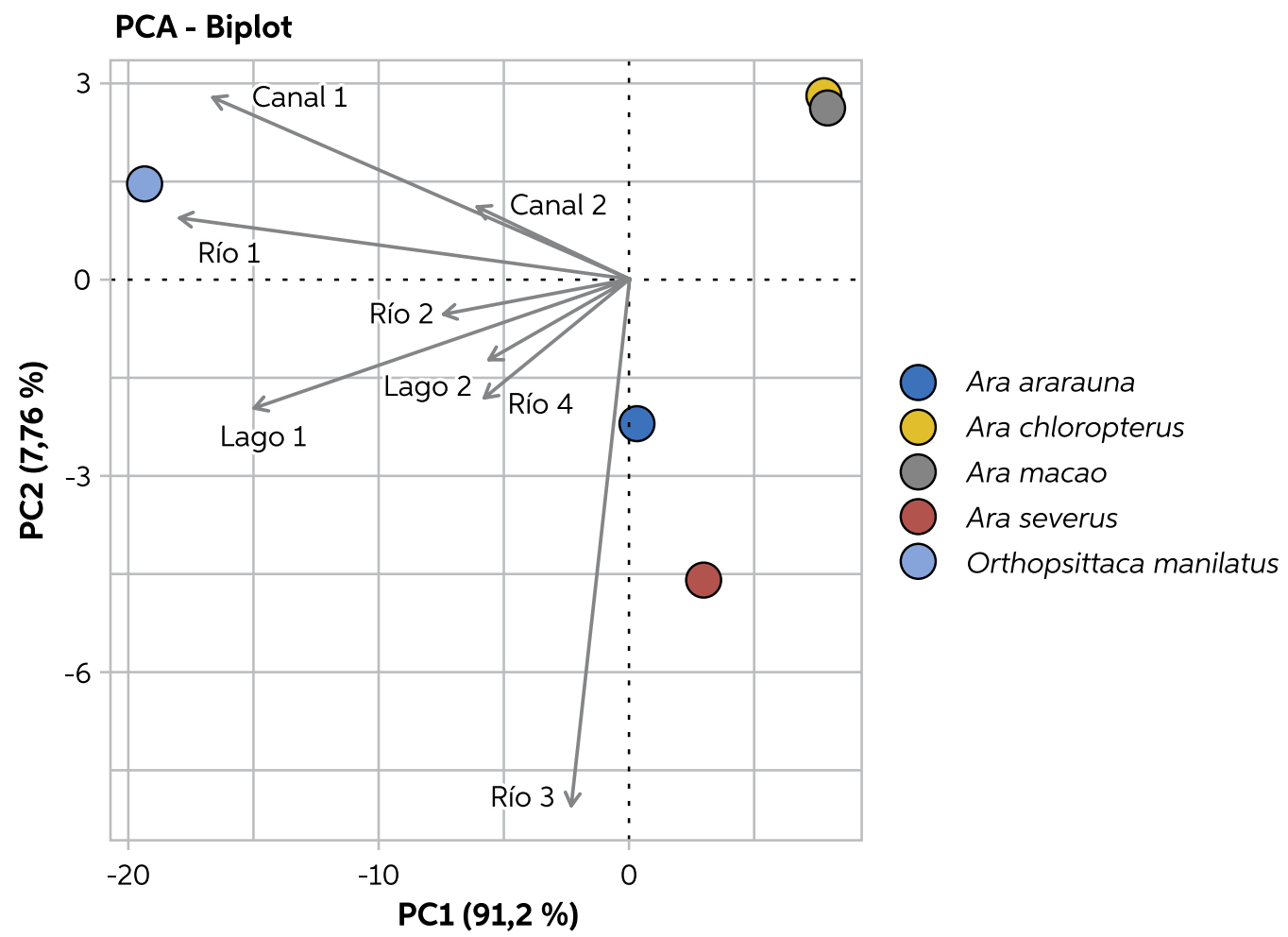

Figura 3. Análisis de Componentes Principales de covarianza mostrando la similitud entre guacamayos y la causa de sus diferencias (vectores).

Tabla 1. Relaciones de las abundancias entre las especies de guacamayos. Los valores de la diagonal inferior indican el coeficiente de correlación de Pearson y los valores de la diagonal superior indican la probabilidad o p-valor (p). El valor de la corrección de Bonferroni es $p \leq 0,0125$, por ello solo los valores por debajo de este aceptan la hipótesis alterna o se consideran como significativos $(*)$.

\begin{tabular}{cccccc}
\hline Especies & Ara ararauna & $\begin{array}{c}\text { Ara } \\
\text { chloropterus }\end{array}$ & Ara macao & Ara severus & $\begin{array}{c}\text { Orthopsittaca } \\
\text { manilatus }\end{array}$ \\
\hline Ara ararauna & & 0,300 & 0,146 & $0,012^{*}$ & 0,111 \\
Ara chloropterus & $-0,326$ & & 0,013 & $0,003^{*}$ & 1,000 \\
Ara macao & 0,446 & $-0,692$ & & 0,013 & 0,369 \\
Ara severus & 0,695 & $-0,771$ & 0,689 & & 0,323 \\
Orthopsittaca manilatus & 0,484 & 0,000 & $-0,285$ & 0,312 & \\
\hline
\end{tabular}


de abundancia en ninguno de los sectores por cada transecto. Es decir, se evidenció que la abundancia de 0 . manilatus estuvo relacionada a su mayor abundancia en los siguientes vectores: Canal 1, Lago 1 y Río 1, en el sector Tacshacocha. Mientras que $A$. ararauna y $A$. severus sólo fueron abundantes en Río 3, situado en el sector Huishtococha.

La abundancia de los guacamayos no solamente estuvo influenciada por los sectores y los transectos, sino que es probable que exista competencia interespecífica. Basado en la correlación de Pearson con corrección de Bonferroni (Tabla 1), hubo una relación negativa entre $A$. severus y $A$. chloropterus ( $\mathrm{r}=-0,771$; $p=0,003$ ), y una relación positiva entre $A$. severus y $A$. ararauna $(\mathrm{r}=0,695 ; p=0,012)$. Es decir, existen relaciones entre especies medianamente abundantes y una rara, así como también relación entre una especie muy abundante con otra medianamente abundante.

La abundancia de guacamayos no fue homogénea durante todo el año, sino que fluctúa según la estación (Figura 4). A. ararauna fue la más abundante desde finales de la época de creciente (abril) hasta octubre, mes donde la época de vaciante está en su máxima expresión. Ara macao y $A$. severus presentaron su máxima abundancia en la época con mayor nivel de inundación. Sin embargo, A. severus tuvo un segundo pico de abundancia en el mes de agosto, es decir, en la transición de época creciente a vaciante. A. chloropterus tuvo un pico máximo de abundancia durante la vaciante en el mes de octubre y una mínima durante el mes de mayo, donde la época de creciente está en su máxima expresión. O. manilatus fue abundante entre los meses de junio y julio, época cuando inicia el descenso del nivel de las aguas. Los resultados indican que las máximas abundancias difieren entre las especies de guacamayos. Algunos pueden coincidir parcialmente como el caso de $A$. severus y $A$. macao, así como $A$. ararauna y $O$. manilatus.

La abundancia de guacamayos fue fluctuante en el tiempo, es decir, ninguna de las especies mostró abundancias estables año tras año. Asimismo, estas fluctuaciones no desarrollaron declinaciones poblacionales desde el 2006 al 2015 (una década de análisis) y fueron estables en el tiempo (Figura 5). Ninguna de las especies del género Ara tuvieron una declinación o incremento poblacional significativo $(p>0,05)$. La tendencia de estas especies permaneció estable durante la última década. La abundancia de $A$. ararauna tuvo picos máximos en los años 2009 y 2011, y el pico más bajo durante el 2007. En el caso de $A$. chloropterus, la abundancia tuvo un pico máximo en el año 2008, y el pico más bajo durante el 2006 y 2009. Ara macao presentó picos máximos de abundancia en el año 2008 y 2009, y el pico más bajo durante el 2006 y 2013. Ara severus tuvo su pico máximo de abundancia en el año 2009, y el pico más bajo durante el 2007 y 2008. Sin embargo, Orthopsittaca manilatus fue la única especie que tuvo tendencia poblacional a incrementarse $(p=0,02)$. Antes del 2010 la abundancia fue muy baja, después de este año la abundancia se incrementó y tuvo su pico máximo en el año 2012, pero no disminuyó sustancialmente desde aquel año.

El análisis de componentes principales mostró dos grandes grupos, uno antes y otro después de la vaciante del 2010 (ANOSIM, $r=0,644$; $p=0,01)$. La comunidad de las especies de $A$. severus y $A$. ararauna fueron dominantes antes de la máxima vaciante del 2010; sin embargo, después del evento climático solamente la composición de $O$. manilatus fue abundante. Los resultados de la tendencia poblacional, reflejan el incremento poblacional significativo de 0 . manilatus, que estuvo vinculado con la 


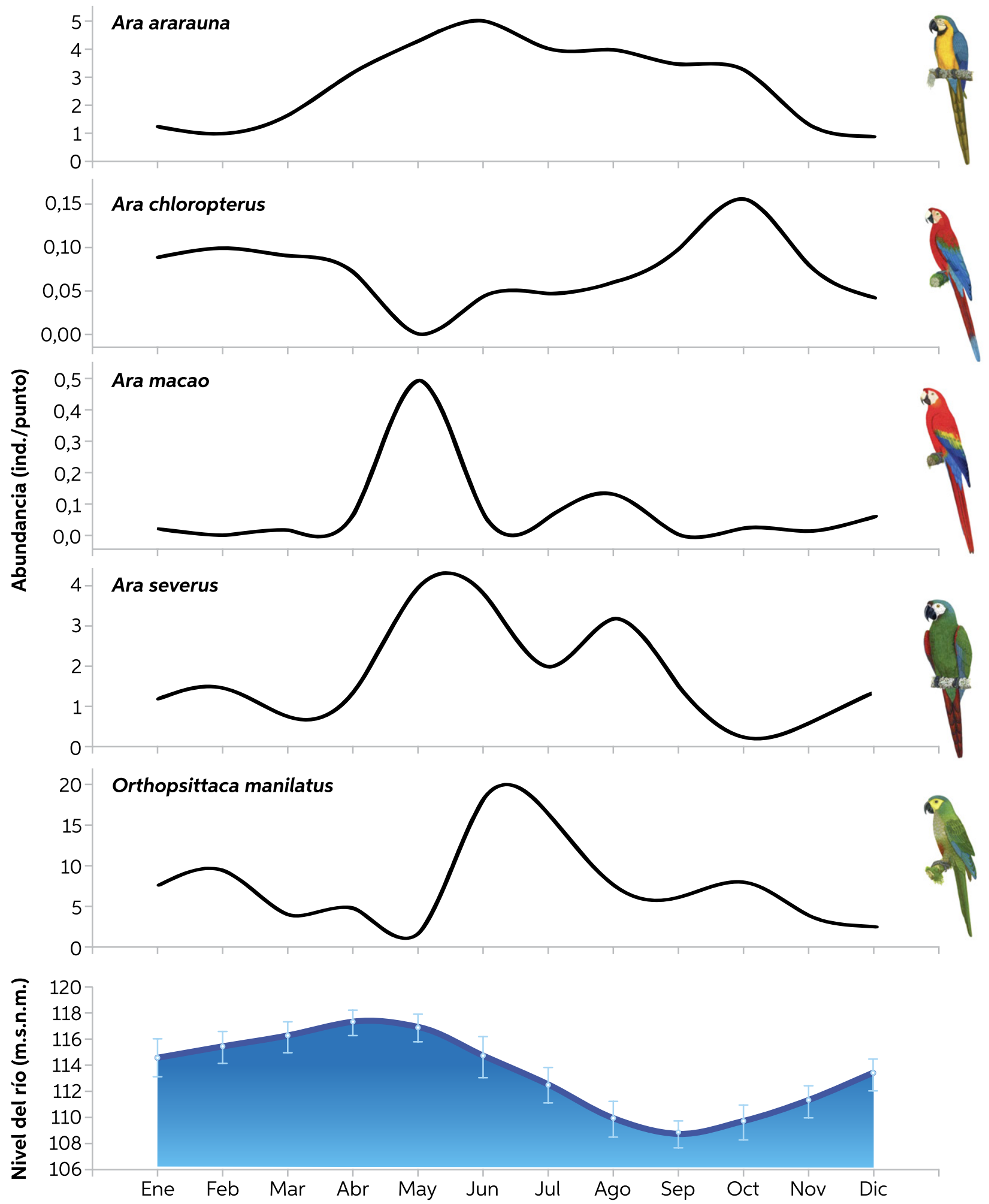

Figura 4. Estacionalidad de la abundancia (ind./punto) de las especies de guacamayos en la cuenca media del río Samiria (Loreto, Perú). La línea azul indica el promedio mensual del nivel del río y las líneas celestes indican la desviación estándar. 

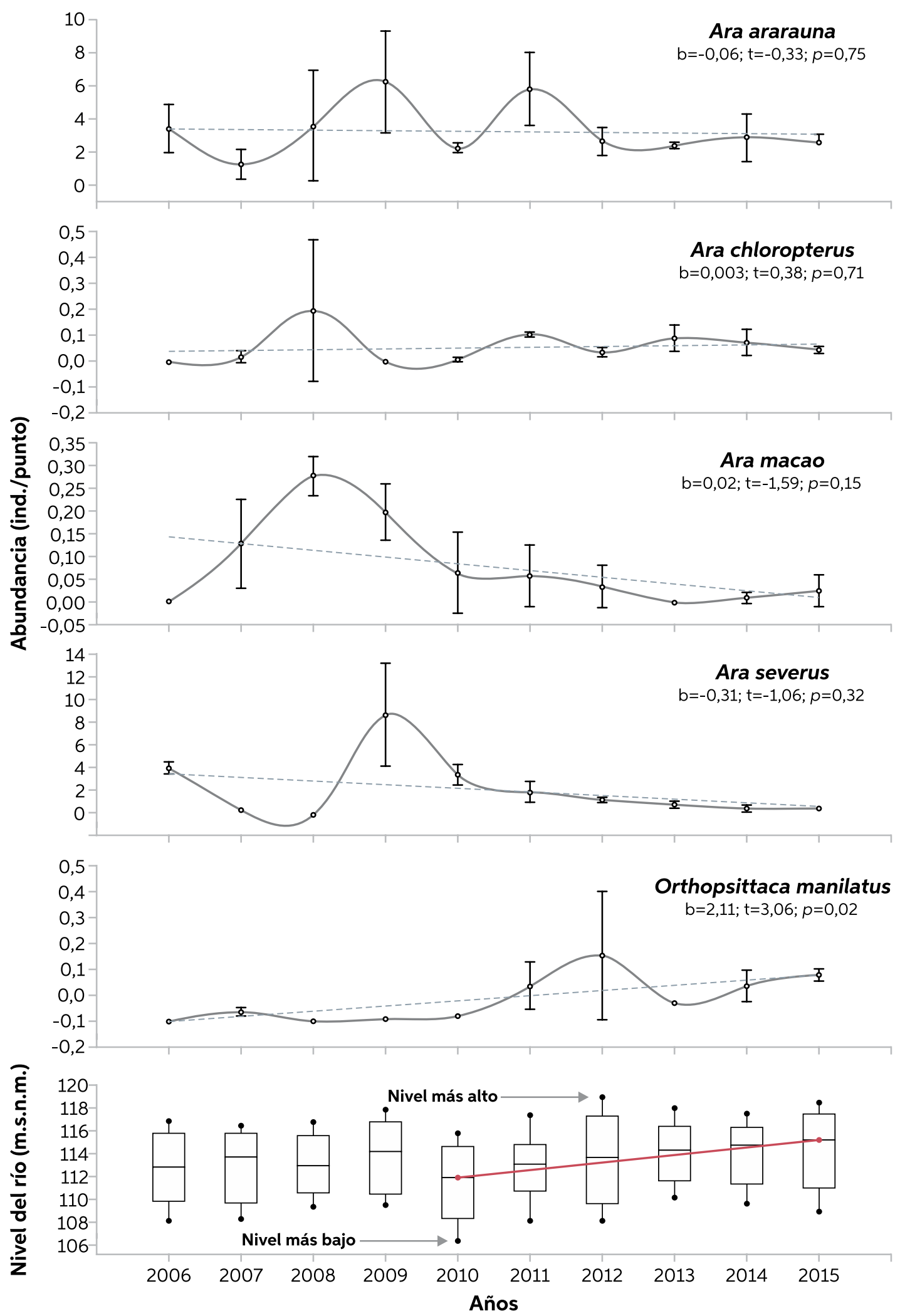

Figura 5. Tendencia poblacional de las especies de guacamayos desde el 2005 al 2016 en la cuenca media del río Samiria. 


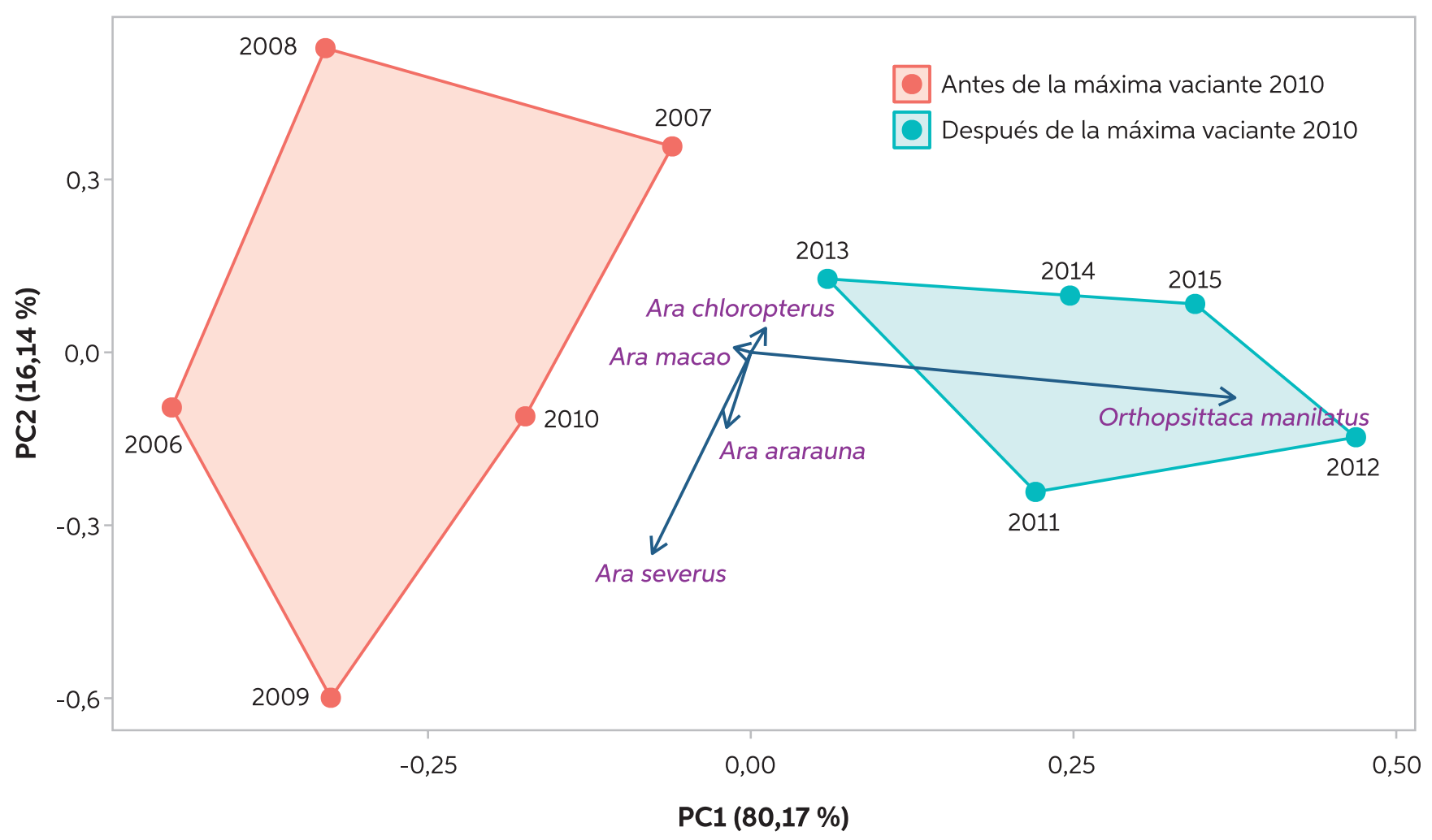

Figura 6. Análisis de Componentes Principales de covarianza mostrando dos grupos antes y después del 2010. Las flechas azules indican las especies más abundantes en cada grupo.

disminución de la abundancia de las dos especies antes abundantes, posterior a la máxima vaciante del 2010 (Figura 6).

\section{DISCUSIÓN}

Actualmente, existe cinco especies de guacamayos en la Reserva Nacional Pacaya Samiria (Bodmer et al., 2014), de las cuales todas fueron registradas y estudiadas en este trabajo de investigación. En la cuenca media del río Samiria, las especies de guacamayos presentaron diferencias significativas en cuanto a la abundancia, similar a lo registrado por Bodmer et al. (2011) en distintas zonas de la Amazonía peruana. El sector Tacshacocha fue la zona con mayor cantidad de avistamientos de individuos en comparación a la zona de Huishtococha, mientras que Bodmer et al. (2006), mencionan que existe mayor presencia de guacamayos en el área adyacente a la zona de Shiringal, en la cuenca baja del río Samiria. Estos contrastes en los avistamientos, se debió a que, en este estudio no se incluyeron individuos vistos fuera de lo horarios de observación, en varias ocasiones los esfuerzos de muestreo fueron interrumpidos por condiciones climáticas a escala local, y por la presencia del mosaico heterogéneo de llanuras meándricas, aguajales y pantano arbóreo del área de estudio (MINAM, 2015) que proporcionan recursos alimenticios para las especies frugívoras que pueden desplazarse dentro y fuera de las áreas de tierra firme en periodos cortos de tiempo.

Orthopsittaca manilatus, Ara ararauna y A. severus fueron las especies con el mayor 
número de avistamientos, similar a lo reportado por Sánchez (2013) y Bodmer et al. (2014). Es necesario aclarar que mostramos sólo correlaciones, y no análisis de causa-efecto, lo cual pertenece a un análisis de regresión, como es mostrado en Bodmer et al. (2006), que afirman que el tipo de vegetación como los aguajales y várzeas, influye en la abundancia. Esto se ve reflejado por la disponibilidad de recursos y lugares ideales para postura como lo menciona Berkunsky et al. (2016). Esta influencia no está presente en A. macao y A. chloropterus, que se reproducen principalmente en bosques de tierra firme y escasamente en aguajales (Bodmer, 2006), y se desplazan en pequeños grupos familiares o en parejas. Sin embargo, nuestros resultados sugieren que se deben realizar estudios ecológicos estacionales más detallados para mostrar competencias intra e interespecíficas. Esto se atribuye a la premisa observada basada en la teoría del nicho, donde especies similares en términos de trazas funcionales sufren competencia que afecta directamente su abundancia (MacArthur \& Levis, 1964).

Nuestros resultados concuerdan con Bodmer et al. (2011) y Bodmer et al. (2014), que muestran la tendencia poblacional de las especies de guacamayos como fluctuantes. Tal es así que, tanto Ara severus como Orthopsittaca manilatus tuvieron cambios significativos en cuanto a su abundancia, por ejemplo, la tendencia de $O$. manilatus es a incrementar $(\mathrm{b}=2,11 ; \mathrm{t}=3,06$; $p=0,02)$. Sánchez (2013) indicó que Ara ararauna y $A$. severus mantuvieron una tendencia estable, y tanto A. chloropterus como A. macao presentaron una ligera tendencia a incrementar. Sin embargo, mostramos que estas especies presentaron una tendencia estable, indicando que ninguna especie está en proceso de declinación. Esto podría estar relacionado con la capacidad de dispersión o migraciones cíclicas de estas especies en épocas de sequía o inundación extrema.
Durante las últimas décadas la cuenca amazónica se ha vuelto más cálido y un poco más húmedo (Skansi et al., 2013) afectando el ciclo hidrológico del Amazonas. Con base en los resultados obtenidos del análisis de similitud (ANOSIM), la sequía o máxima vaciante ocurrida en 2010 parece haber afectado el ensamble de guacamayos en la cuenca media del río Samiria. $A$. severus y $A$. ararauna fueron las especies dominantes antes de la máxima vaciante del 2010, mientras que O. manilatus, incrementó su población posterior a la máxima vaciante del 2010. Esto apoya una de las mayores premisas de la teoría de dominio (Ketterson \& Nolan, 1976) que establece que aves de mayor porte superan a las más jóvenes y las de menor tamaño en tiempo de condiciones ambientales adversas, obligando a migrar a otros sitios en búsqueda de alimento o nidos en determinadas épocas del año. Este patrón de tendencia está relacionado con la variación fenológica estacional (van Schaik et al., 1993) influida por el nivel anual del río y eventos climáticos ocurridos durante la máxima vaciante en el 2010 e inundaciones extremas del 2012 (Marengo \& Espinoza, 2015) que afectaron la biodiversidad en la Amazonía.

De acuerdo con Bodmer et al. (2017), es conveniente llevar a cabo un monitoreo a largo plazo, en el mismo lugar y en las mismas épocas del año, considerando la variación temporal del muestreo (esfuerzo de muestreo) (Ojasti, 2000) para asegurar que las tendencias observadas sean robustas y no un artefacto resultante de las fluctuaciones normales de los factores ambientales o la estacionalidad. Estos fenómenos podrían afectar a largo plazo la estructura de los bosques inundables y disponibilidad de alimento (Bodmer et al., 2014).

Según la IUCN (2020), las cinco especies registradas están en la categoría de preocupación menor (NT), Orthopsittaca manilatus muestra una tendencia estable mientras que las otras cuatro 
especies de guacamayos están disminuyendo. Basado en los resultados del presente estudio, ninguna de las especies de guacamayos está en proceso de declinación en la cuenca media de la Reserva Nacional Pacaya Samiria y mantienen su continuidad en el tiempo con resultados positivos.

En conclusión, nuestros resultados revelan la importancia de considerar estudios a largo plazo para mostrar fluctuaciones poblacionales producidas a lo largo del tiempo por varios eventos climáticos extremos. Algunas especies mostraron plasticidad adaptativa en sus tendencias y otras aumentaron su población debido a los eventos ocurridos entre el 2009 y 2012. En efecto, el régimen de lluvias influye en el nivel de agua del río, afectando la disponibilidad de alimentos (Iñigo-Elías, 1999) y frutos de aguaje (variables no consideradas), estas características ecológicas, estarían influenciando con la época reproductiva y la edad del inicio de vuelo de cada especie de guacamayo en el ámbito de la Reserva Nacional Pacaya Samiria.

Estos factores no se consideraron como variables explicativas, y sugerimos que se integren para futuras investigaciones, tales como el uso de modelos de nicho ecológico que permitiría abordar mejor los cambios en distribución asociados al cambio climático (Monterrubio-Rico et al., 2016) y monitoreo sistemático (Voughan, 2019) sobre una percepción ambiental para la conservación de Psitácidos en toda la cuenca de la Reserva Nacional Pacaya Samiria.

\section{AGRADECIMIENTOS}

Al Blgo. Pablo Puertas por su apoyo en el campo y orientación durante la redacción. Al Ing. Marco Antonio Paredes Riveros del Servicio Nacional de Meteorología e Hidrología del Perú, por proporcionar información sobre el nivel del río
Amazonas. Al Dr. Jack Shutt, Emilio Payo y Pedro Pérez Peña, por sus comentarios y sugerencias en la redacción del manuscrito. A los guías locales de campo y al personal del SERNANP de la Reserva Nacional Pacaya Samiria, a las comunidades Cocama del río Samiria. El financiamiento para este estudio fue proporcionado por FundAmazonia, WCS, Earthwatch Institute, Operation Wallacea, Operations Earth y AmazonEco.

\section{REFERENCIAS BIBLIOGRÁFICAS}

Bayley, P.B.; Vásquez, R.P.; Ghersi, P.F.; Soini, P.; Pinedo, P.M. 1991. Environmental Review of the Pacaya-Samíria Reserve in Peru and assessment of project (527-0341). The Nature Conservancy, Iquitos. 81pp.

Berkunsky, I.; Cepeda, R.; Marinelli, C.; Simoy, V.; Daniele, G.; Kacoliris, F.P.; Luque, J.A.; Gandoy, F.A.; Aramburú, R.; Gilardi, J.D. 2016. Occupancy and abundance of large macaws in the Beni savannahs, Bolivia. Oryx, 50: 113120. DOI: https://doi.org/10.1017/S003060 5314000258

Bodmer, R.; Puertas, P.; Pérez, P.; Ríos, C.; Dosantos, A.; Recharte, M.; Flores, W.; Arévalo, F.; Ruck, L.; Antúnez, M.; Valverde, Z.; Moya, L.; Freitas, G. 2006. Estado actual de las especies paisajísticas de fauna silvestre y del monitoreo a grupos de manejo de la cuenca del Samiria -RNPS. Proyecto: Conservación de la vida silvestre en la Amazonía Peruana de Loreto. Wildlife Conservation Society, Iquitos. 319pp.

Bodmer, R.; Puertas, P.E.; Antúnez, M.S.; Fang, T.G.; Pérez, P.E. 2011. Monitoreo de especies indicadoras para evaluar el impacto del cambio climático en la cuenca del Samiria, Reserva Nacional Pacaya Samiria. Reporte anual 20102011. 207pp.

Bodmer, R.; Fang, T.; Puertas, P.; Antúnez, M.; Chota, K.; Bodmer, W. 2014. Cambio climático 
y fauna silvestre en la Amazonía Peruana. Impacto de la sequía e inundaciones intensas en la Reserva Nacional Pacaya Samiria. Fundación Latinoamericana para el Trópico Amazónico (Fundamazonia). Wust Ediciones. 60pp.

Bodmer, R.; Fang, T.; Antunez, M.; Puertas, P.; Chota, K.; Pittet, M.; Kirkland, M.; Walkey, M.; Rios, C., Perez-Peña, P.; Mayor, P.; Zegarra, J.; Dorcherty, E. 2017. Impact of recent climate fluctuations on biodiversity and people in flooded forests of the Peruvian Amazon. In: Rodríguez, L.; Anderson, I. (Eds.). The Lima Declaration on Biodiversity and Climate Change: Contributions from science to policy for sustainable development. Secretariat of the Convention on Biological, Montreal, Technical Series No.89. p. 81-89.

CITES (Convención sobre el Comercio Internacional de Especies Amenazadas de Fauna y Flora Silvestres). 2020. Apéndice I, II y III. (https://www.cites.org/esp). Acceso: 10/09/2020.

Costa, F.; Zuanon, J.; Baccaro, F.; Schietti, J.; Menger, J.; Souza, J.L.P.; Borba, G.C.; Esteban, E.J.L.; Bertin, V.M.; Gerolamo, C.S.; Nogueira, A.; Castilho, C.V. 2020. Effects of climate change on central amazonian forests: a two decades synthesis of monitoring tropical biodiversity. Oecologia Australis, 24(2): 317-335. DOI: https://doi.org/10.4257/oeco.2020.2402.07

Espinoza, J.; Guimberteau, M.; Guyot, J.L.; Lavado, W.J.R.; Santini, W. 2014. Eventos hidrológicos extremos en la cuenca amazónica peruana: presente y futuro. A Gr'egoire (Ed.). El Perú frente al cambio climático: resultados de investigaciones franco-peruanas. Lima, Perú: IRD, p. 47-58.

Gloor, M.; Brienen, R.J.W.; Galbraith, D.; Feldpausch, T.R.; Schöngart, J.; Guyot, J.L.; Espinoza, J.C.; Lloyd, J.; Phillips, O.L. 2013. Intensification of the Amazon hydrological cycle over the last two decades. Geophysical Research
Letters, 40(9): 1729-1733. DOI: https://doi. org/10.1002/grl.50377

Hosein, A.; Narang, D.S.; Rostant, L.; Haile, A. 2017. The abundance of Red-bellied Macaws (Orthopsittaca manilatus) and Orange-winged Parrots (Amazona amazonica) in relation to fruiting Moriche palms (Mauritia flexuosa) at the Aripo savannas, Trinidad. Revista Brasileira de Ornitologia, 25(1): 40-46. DOI: https://doi. org/10.1007/BF03544375

Iñigo-Elías E. 1999. Las guacamayas verde y escarlata en México. Biodiversitas 25:7-11.

IUCN (The International Union for Conservation of Nature). 2020. The IUCN red list of threatened species. Version 2020-1. (www. iucnredlist.org). Acceso: 05/05/2020.

Ketterson, E. D.; V. Nolan. 1976. Geographic variation and its climatic correlates in the sex ratio of eastern-wintering Dark-Eyed Juncos (Uunco hyemalis). Ecology, 57:679-693. DOI: https://doi.org/10.2307/1936182

MacArthur, R.; Levins, R. 1964. Competition, habitat selection, and character displacement in a patchy environment. Proceedings of the National Academy of Sciences of the United States of America, 51(6): 1207. DOI: https:// doi.org/10.1073/pnas.51.6.1207

Marengo, J.A.; Espinoza, J.C. 2016. Extreme seasonal droughts and floods in Amazonia: causes, trends and impacts. International Journal of Climatology, 36(3): 1033-1050. DOI: https://doi.org/10.1002/joc.4420

MINAM (Ministerio del Ambiente). 2015. Mapa nacional de cobertura vegetal: memoria descriptiva. Ministerio del Ambiente, Lima. 100pp.

Monterrubio-Rico T.C.; Charre-Medellín, J.F.; Pacheco-Figueroa, C.; Arriaga-Weiss, S.; Valdez-Leal, J.D.; Cancino-Murillo, R.; EscalonaSegura, G.; Bonilla-Ruz, C.; Rubio-Rocha, Y. 2016. Distribución potencial histórica y contemporánea de la familia Psittacidae en 
México. Revista Mexicana de Biodiversidad, 87(3): 1103-1117. DOI: https://doi.org/ 10.1016/j.rmb.2016.06.004

Ojasti, J. 2000. Manejo de Fauna Neotropical. U.S: F Dallmeier (Ed.). Smithsonian. Institution, U.S. Fish \& Wildlife Service, WWF, UNESCO. 290pp.

Plenge, M.A. 2020. List of the birds of Peru / Lista de las aves del Perú. Unión de Ornitólogos del Perú: (https://sites.google.com/site/ boletinunop/checklist). Acceso: 24/10/2020.

RStudio Team. 2019. RStudio: integrated development for R. RStudio, (https://www. rstudio.com). Acceso: 19/12/2019.

Sánchez, D. 2013. Algunos aspectos poblacionales de Ara spp. (Lacépede 1799) "Guacamayos" en el sector inferior del río Samiria en época de vaciante - Reserva Nacional Pacaya Samiria, Loreto, Perú. Tesis Pregrado. Universidad Nacional de la Amazonía Peruana, Facultad de Ciencias Biológicas. Iquitos, Perú. 78pp.

SENAMHI (Servicio Nacional de Meteorología e Hidrología del Perú). 2020. Procedimientos para adquirir información. Lima, Perú. (http:// www.senamhi.gob.pe/?p=0612). $\quad$ Acceso: 21/01/2020.

Silva, P.A.; Melo, C.D. 2015. Variação na abundância do maracanã-do-buriti Orthopsittaca manilatus (Psittacidae) e produção de frutos no buriti Mauritia flexuosa (Arecaceae). Ambiência, 11(3): 611-628. DOI: https://doi. org/10.5935/ambiencia.2015.03.07

Skansi, M.; Brunet, M.; Sigró, J.; Aguilar, E.; Groening, J.A.A; Betancur, O.J.; Geier, Y.R.C.; Corre-Amaya, R.L.; Jácome, H; Ramos, A.M.; Rojas, C.O.; Pasten, A.M.; Mitro, S.S.; Jímenez, C.V.; Martínez, R.; Alexander, L.V.; Jones P.D. 2013. Warming and wetting signals emerging from analysis of changes in climate extreme indices over South America. Global and Planetary Change 100:295-307. DOI: https:// doi.org/10.1016/j.gloplacha.2012.11.004

Systat Inc. 2017. Sigmaplot for Windows, Version 14 (https://systatsoftware.com/products/ sigmaplot/). Acceso: 29/10/2017.

van Schaik, C.P.; Terborgh, J.W.; Wright, S.J.1993. The phenology of tropical forests: adaptive significance and consequences for primary consumers. Annual Review of Ecology and Systematics, 24(1): 353-377. DOI: https://doi. org/10.1146/annurev.es.24.110193.002033

Zulkafli, Z.; Buytaert, W.; Manz, B.; Rosas, C.V.; Willems, P.; Lavado-Casimiro, W.; Guyot J.L.; Santini, W. 2016. Projected increases in the annual flood pulse of the Western Amazon. Environmental Research Letters, 11(1): 014013. DOI: https://doi. org/10.1088/1748-9326/11/1/014013

Recibido: 11 de setiembre de 2020 Aceptado para publicación: 22 de octubre de 2020 